\title{
DYNAMIC COMPUTATION AND DESIGN OF A WORLD RECORD DEATH-RIDE
}

\author{
Vincent Denoël ${ }^{1}$, Thomas Canor ${ }^{2}$, Johan Demanet ${ }^{3}$ \\ ${ }^{1}$ Structural Engineering Division, University of Liege \\ 1, Chemin des Chevreuils, B52/3, 4000 Liege, Belgium \\ e-mail: v.denoel@ulg.ac.be \\ ${ }^{2}$ National Fund for Scientific Research, Structural Engineering Division, University of Liege \\ 1, Chemin des Chevreuils, B52/3, 4000 Liege, Belgium \\ e-mail: t.canor@ulg.ac.be \\ ${ }^{3}$ Regional Fire Brigade, GRIMP (Groupe de Reconnaissance et d'Intervention en Milieu Prilleux) \\ 12, Rue des Bourgeois, 5000, Namur, Belgium
}

Keywords: Instructions, ECCOMAS Thematic Conference, Structural Dynamics, Earthquake Engineering, Proceedings.

Abstract. A death-ride is a long cable tensioned between two dead ends. Its design should offer a comfortable and/or enjoyable journey to the riders, within the strength limits of the material. Actually, the tensioning operation is usually subtle as a too slack cable with a sagging area would not allow finish the race, while a too taut cable could produce excessive stresses in the cable. The paper presents the design methodology and the dynamic model that were used for the design of a world-record $1.2 \mathrm{~km}$ long death-ride, with an only 10-mm diameter. 

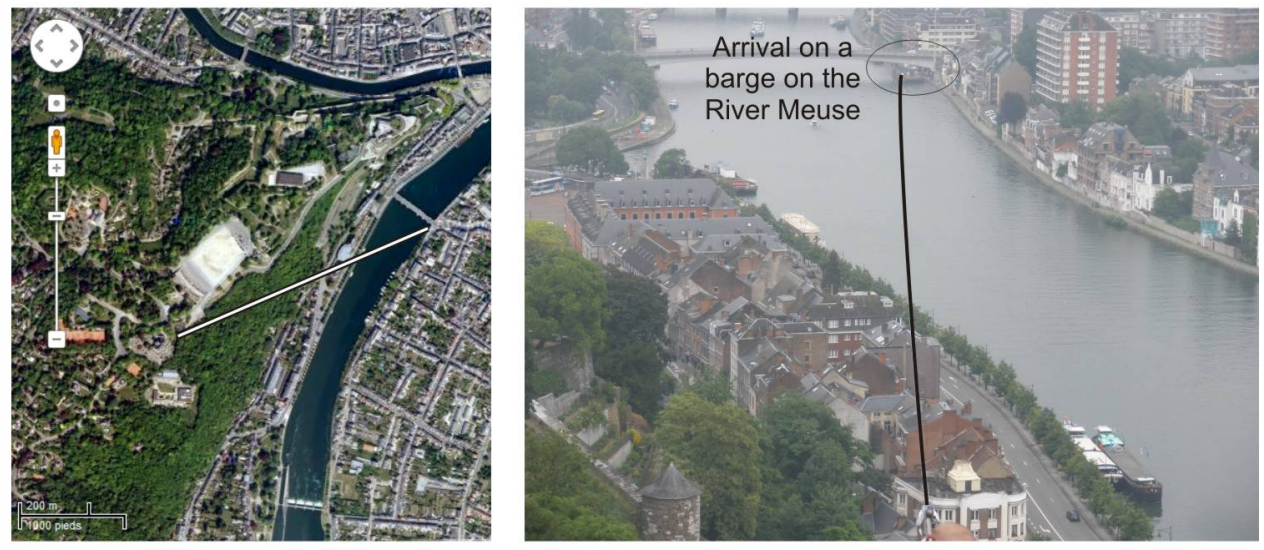

Figure 1: Location of the GrimpDay event, Namur-Jambes (Belgium) - June 2010.

\section{Introduction}

A death-ride is a long cable anchored between two dead ends, allowing the crossing of wide valleys. Besides mobility purposes, it also proves to be useful in rescue operations, or even sometimes simply for leisure.This paper reports the design of a world record death-ride, approx. $1200 \mathrm{~m}$ long in urban area. The cable was temporarily installed in June 2010 for an international competition organized by the special intervention group of the national fire brigade [13]. Figure 1 indicates the location of the event. In the heart of the city of Namur, Belgium, the cable planning was purposed to connect the citadel located on a hill to a barge in the river Meuse $\Delta y=112 \mathrm{~m}$ below, and with a horizontal offset $\Delta x=1163 \mathrm{~m}$.

From a serviceability viewpoint, a death-ride design should offer a safe, comfortable and also sometimes enjoyable journey to the riders, which is typically expressed in terms of maximum velocity. From a strength viewpoint, a death-ride should be designed in order to safely withstand the cable self-weight, wind forces, the rider's load and at last but not least, the initial tension in the cable.

The initial tension in the cable has to be tuned accurately. Indeed, in case of a loose cable, the rider may be stopped in the in-span sagging area, i.e. suspended above the river Meuse in this case. Conversely a too tight cable would generate large stresses but also a large velocity at the end of the ride, which could make it unsafe, for the rider has to be stopped. The design of a death-ride is a tradeoff between all these aspects.

There is a limitation in the acceptable length of a death-ride. Indeed, super long death-rides require large initial tension in order to maintain an acceptable deflection at mid-span [9, 8], and to limit the low-frequency oscillations resulting from wind buffeting [2, 11]. This large initial tension can only be withstood by the cable if it has a sufficient diameter, which in turn increases the distributed weight, reduces the natural frequencies and further necessitates the initial tension force to be increased. A peculiarity of the death-ride considered in this paper, which makes it thus remarkable in several aspects, is the limitation of the cable cross-section to an only $10-\mathrm{mm}$ diameter, a constraint fixed in the very first stages of this project for compatibility reasons with some existing riding equipment.

Cables are usually modeled as structural elements without bending stiffness. The only way they can withstand external transverse forces is to find equilibrium in a largely displaced configuration [7]. While the model considered in this paper is limited to a planar representation 
[4], some tridimensional models offer accurate modeling of the complex nonlinear vibrations of cable in both the vertical and the horizontal directions [7].

The zero bending stiffness assumption is actually defeated in the short zones near discontinuities such as clamped anchorages or concentrated forces. In this latter case, which is typically met as the rider travels along the cable, there exists a short zone on each side of the traveling force where the cable inclination varies rapidly. These short boundary layers [10] results in the relative inclination in the cable, between both sides of the force, as seen from a far-field viewpoint. Analytical methods are usually developed in order to avoid the difficult numerical modeling of these boundary layers [3, 5]; because this model is mainly devoted to be numerical, this feature is disregarded in this work. It is thus assumed that stresses resulting from this high-gradient bending is negligible with respect to tensile stresses.

The dynamic loading of structures under moving loads is extensively studied in [6]. In particular, the vibrations of a string subject to a moving force is a well-established theory. A major difference with the death-ride problem however, is that the traveling velocity of the rider is a priori unknown as it results from the interaction between the cable deflection and the rolling pulley. This aspect is further detailed in the next section.

\section{The Death-Ride Model}

\subsection{The cable model}

The cable is represented with a 2-D chain-like model composed of large displacement truss elements written with a corotational updated framework [14]. The cable is thus discretised as a set of $n$ finite elements, each of them connecting two of the $n+1$ nodes of the model, see Fig. 2-a. In the initial configuration, nodes are uniformly distributed between two end points and are referenced with their position $\left(x_{i}, y_{i}\right)$ in a fixed reference frame $\left(\mathbf{e}_{x}, \mathbf{e}_{y}\right)$. For simplicity in the notations, these coordinates are collected in a $(2 n+2) \times 1$ vector $\mathbf{x}$ concatenating $\mathbf{x}-$ and y-positions, alternatively, for each of the $n+1$ nodes.

Elements are defined by two nodes and a uniaxial displacement field in the corotational frame. In the initial deformation, their description is complemented by an initial length and inclination given by

$$
\ell_{i}=\left[\left(x_{i+1}-x_{i}\right)^{2}+\left(y_{i+1}-y_{i}\right)^{2}\right]^{1 / 2} \quad ; \quad \alpha_{i}=\arctan \frac{y_{i+1}-y_{i}}{x_{i+1}-x_{i}} .
$$

The displacement field $\mathbf{u}(t)$ collecting the $\mathrm{x}$ - and $\mathrm{y}$ - components, in the reference frame, of the nodal displacements, provides the nodal coordinates $\mathbf{x}^{\prime}(t)$ in the deformed configuration, see Fig. 2-b,

$$
\mathbf{x}^{\prime}(t)=\mathbf{x}+\mathbf{u}(t)
$$

Nodal velocities and accelerations are readily obtained as the first and second time derivative of (2). The kinematics of each element are fully determined by the location of its two end nodes. Actually, the length and the inclination of each truss element in the deformed configuration,

$$
\ell_{i}^{\prime}=\left[\left(x_{i+1}^{\prime}-x_{i}^{\prime}\right)^{2}+\left(y_{i+1}^{\prime}-y_{i}^{\prime}\right)^{2}\right]^{1 / 2} \quad ; \quad \alpha_{i}^{\prime}=\arctan \frac{y_{i+1}^{\prime}-y_{i}^{\prime}}{x_{i+1}^{\prime}-x_{i}^{\prime}}
$$

allow estimation of the internal forces with 

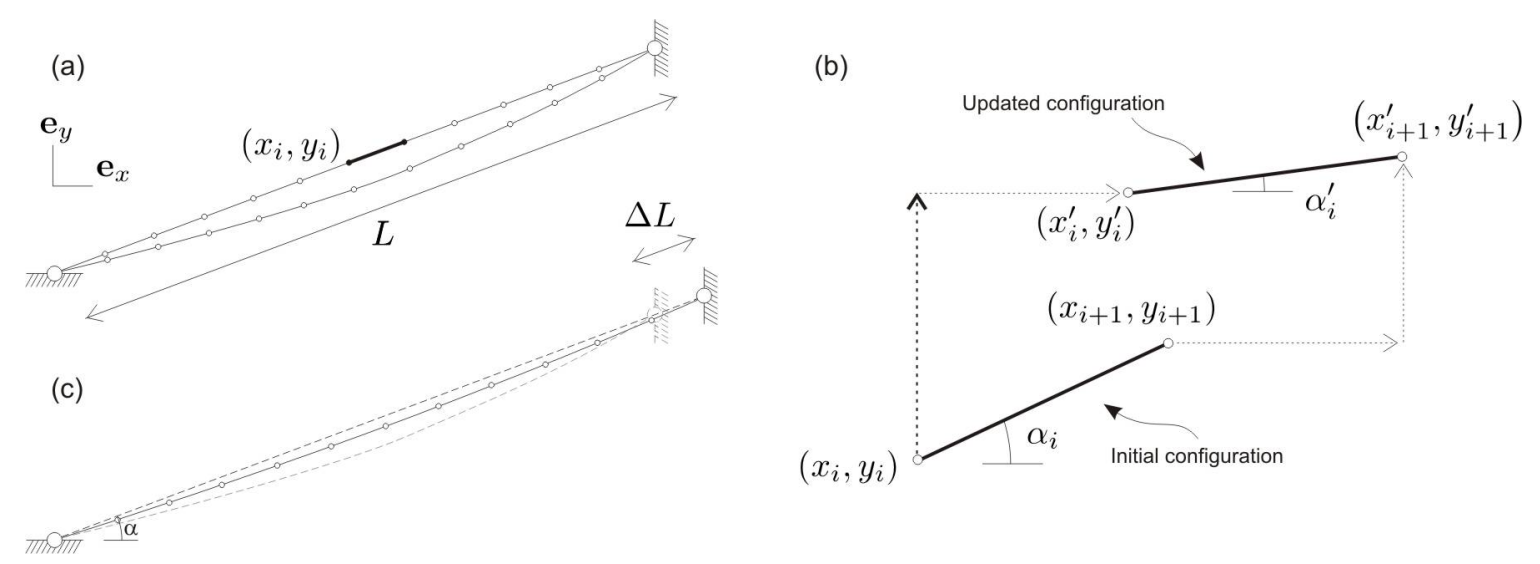

Figure 2: (a) Mesh of initial configuration and sketch of deformed configuration under self-weight, (b) Kinematics of a finite element, (c) Imposed displacement $\Delta L$ at the top node, in order to generate the initial tension in the cable.

$$
\mathbf{f}_{i}(\mathbf{u})=\mathbf{R}\left[\alpha_{i}^{\prime}(\mathbf{u})\right]\left(\begin{array}{c}
-1 \\
0 \\
1 \\
0
\end{array}\right) \frac{E A\left(\ell_{i}^{\prime}-\ell_{i}\right)}{\ell_{i}}
$$

where $E A$ is the axial rigidity of the element, and $\mathbf{R}[\cdot]$ is a finite magnitude rotation matrix

$$
\mathbf{R}[\varphi]=\left(\begin{array}{cc}
\mathbf{T} & \mathbf{Z} \\
\mathbf{Z} & \mathbf{T}
\end{array}\right) ; \quad \mathbf{T}=\left(\begin{array}{cc}
\cos \varphi & -\sin \varphi \\
\sin \varphi & \cos \varphi
\end{array}\right) ; \quad \mathbf{Z}=\left(\begin{array}{cc}
0 & 0 \\
0 & 0
\end{array}\right)
$$

Localization and assembly of internal forces provide the resultant of internal forces $f$,

$$
\mathbf{f}[\mathbf{u}(t)]=\sum_{i=1}^{n} \mathbf{L}_{i} \mathbf{f}_{i}[\mathbf{u}(t)]
$$

where $\mathbf{L}_{i}$ is a localization matrix, with size $(2 n+2) \times 4$.

The equation of motion reads

$$
\mathbf{M} \ddot{\mathbf{u}}(t)+\mathbf{C} \dot{\mathbf{u}}(t)+\mathbf{f}[\mathbf{u}(t)]=\mathbf{p}(t)+\mathbf{r}(t)
$$

where $\mathrm{p}$ represents the external loads associated with the cable own weight and the traveling rider, $\mathbf{r}$ represents the reaction forces, while $M$ represents the global mass matrix, written in this paper with a consistent approach [14], and $\mathbf{C}$ is a damping matrix constructed with a Rayleigh model, imposing a damping coefficient $\xi$ in the first two modes. In the simulation of the structural dynamic response, this equation is integrated with an explicit formulation of Newmark's algorithm.

The response of the cable-rider system is determined in a two-step procedure.

First, the static deformed configuration of the cable under its own weight and initial tension is determined. This first step is achieved by imposing a displacement $\Delta L$ at the top node of the model, in the direction of the chord, see Fig. 2-c. The magnitude of this displacement is translated into a tension in the cable. In accordance with classical static theory of cable, the horizontal projection $H$ of the internal force is used as a reference throughout this document. In more details, the static deflection of the cable $\mathbf{u}_{s}$ is obtained by solving (7) without the first 


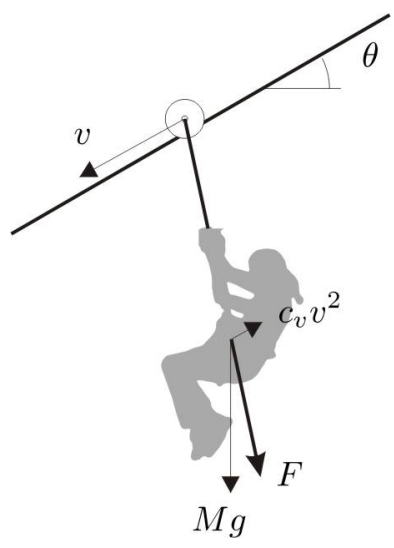

Figure 3: Free-body diagram of the rider (excl. inertial forces).

term - related to inertial forces-, and disregarding the first two and last two equations, as they correspond to unknown reactions. The horizontal force in the cable $H$ is obtained in a postprocessing operation, as the horizontal reaction at the base. Computation of the static deflection $\mathbf{u}_{s}$ as well as the horizontal force in the cable $H$ is repeated for various top displacements $\Delta L$, until the horizontal force maps the target force.

In a second step, the dynamic computation of the cable response is determined, with $\mathbf{u}_{s}$ as initial deformation field. A peculiarity of this problem consists in the determination of what is considered in (7) as external forces p, i.e. those resulting from the coupled cable-rider system. Their expression is discussed in the following section. Apart from this term, the solution of (7) follows classical numerical integration methods.

\subsection{Interaction with the rider}

At the beginning of the computation, the rider leaves the top end of the cable with a zero velocity. Its position along the cable is represented by a supplementary degree-of-freedom $s$, considered as the curvilinear abscissa measured along the cable, from the upper end.

For compatibility reasons, the velocity vector of the pulley rolling on the cable is tangent to the cable supporting the rider, see Figure 3 . Forces acting on the rider are its own weight $M \vec{g}$ and an aerodynamic force $c_{v}|v| \vec{v}$ whose intensity is proportional to the square velocity, as in Bernoulli equation. At the beginning of the ride, the velocity of the rider is rather high, which results in a significantly inclined reaction force $F$; on the opposite, as the rider slows down, its position naturally tends to be below the pulley.

Only the tangential component of the force $F$, i.e. in the direction of the cable, is considered to determine the motion of the rider,

$$
F_{\perp}=M g \sin \theta-c_{v} v^{2} .
$$

Exactly as the motion of a wheel rolling on an inclined plane is determined by the component of the weight parallel to the inclined plane, the acceleration of the rider is determined by

$$
a=g \sin \theta-\frac{c_{v} v^{2}}{M}
$$

In the numerical procedure, starting from the position $s$ and velocity of the rider at the beginning of a time step, the position at the end of a time step is computed under the assumption that the 
acceleration is constant along a time step

$$
v_{t+\Delta t}=v_{t}+a \Delta t \quad ; \quad s_{t+\Delta t}=s_{t}+v_{t} \Delta t+\frac{a}{2} \Delta t^{2}
$$

\subsection{Numerical Algorithm}

The weakly coupled problem is thus solved in a staggered manner, with the algorithm represented by this pseudo-code.

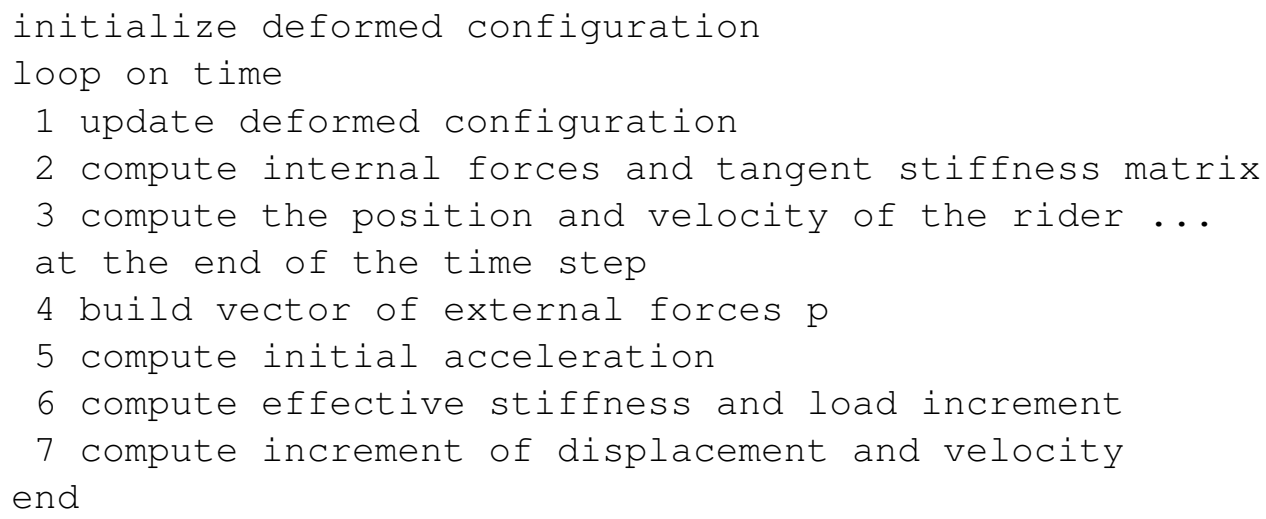

Step 1 consists in the computation of the element lengths $\ell_{i}^{\prime}$ and inclinations $\alpha_{i}^{\prime}$ in the updated deformed configuration, see (3). In step 2, internal forces $f_{t}$ are determined with (6) while the tangent stiffness $\mathbf{K}_{t}$ matrix is obtained by finite difference of internal forces. In step 3, the position and velocity of the rider at the end of the time step are determined by (10). Then the vector of external forces $\mathbf{p}_{t+\Delta t}$ is constructed as a sum of the self-weight forces and the force resulting from the rider. This latter component is mainly composed of zeros, except for the four degrees-of-freedom corresponding to the finite element on which the rider currently is. The magnitude of the force is determined in accordance with the free-body diagram of Fig. 3, and then is dispatched between the four degrees-of-freedom.

The following steps concern the determination of the structural response. In step 5, the acceleration at the beginning of the time step is determined, with

$$
\ddot{\mathbf{u}}_{t}=\mathbf{M}^{-1}\left(\mathbf{p}_{t}+\mathbf{r}_{t}-\mathbf{C}_{t}-\mathbf{f}_{t}\right)
$$

Step 6 consists in determining the effective stiffness and increment of force

$$
\begin{aligned}
\tilde{\mathbf{K}} & =\mathbf{K}_{t}+\frac{3}{\Delta t} \mathbf{C}+\frac{6}{\Delta t^{2}} \mathbf{M} \\
\Delta \mathbf{p} & =\left(\mathbf{p}_{t+\Delta t}-\mathbf{p}_{t}\right)+\mathbf{M}\left(\frac{6}{\Delta t} \dot{\mathbf{u}}_{t}+3 \ddot{\mathbf{u}}_{t}\right)+\mathbf{C}\left(3 \dot{\mathbf{u}}_{t}+\frac{\Delta t}{2} \ddot{\mathbf{u}}_{t}\right)
\end{aligned}
$$

and finally, the increment of structural displacement and velocity are determined by

$$
\begin{aligned}
\Delta \mathbf{u} & =\tilde{\mathbf{K}}^{-1} \Delta \mathbf{p} \\
\Delta \dot{\mathbf{u}} & =\frac{3}{\Delta t} \Delta \mathbf{u}-3 \dot{\mathbf{u}}_{t}-\frac{\Delta t}{2} \ddot{\mathbf{u}}_{t}
\end{aligned}
$$

so that the structural displacement and velocity at the end of the time step are given by adding these increments to the values at the beginning of the time step. 


\begin{tabular}{|c|c|}
\hline$\Delta x=1163 \mathrm{~m}$ & Vertical offset between both ends \\
\hline$\Delta y=112 \mathrm{~m}$ & Horizontal offset between both ends \\
\hline$\xi=0.1 \%$ & Damping ratio \\
\hline$\Omega=77 \mathrm{~mm}^{2}$ & Cable cross section \\
\hline$E=120 \mathrm{GPa}$ & Effective Young modulus \\
\hline$\mu=602 \mathrm{~g} / \mathrm{m}$ & Mass per unit length of the cable \\
\hline$M_{t}=120 \mathrm{~kg}$ & Mass of the rider (plus equipment) \\
\hline$c_{w}=0.74 \mathrm{~N} /(\mathrm{m} / \mathrm{s})^{2}$ & Aerodynamic coefficient \\
\hline
\end{tabular}

Table 1: Numerical data of the problem.
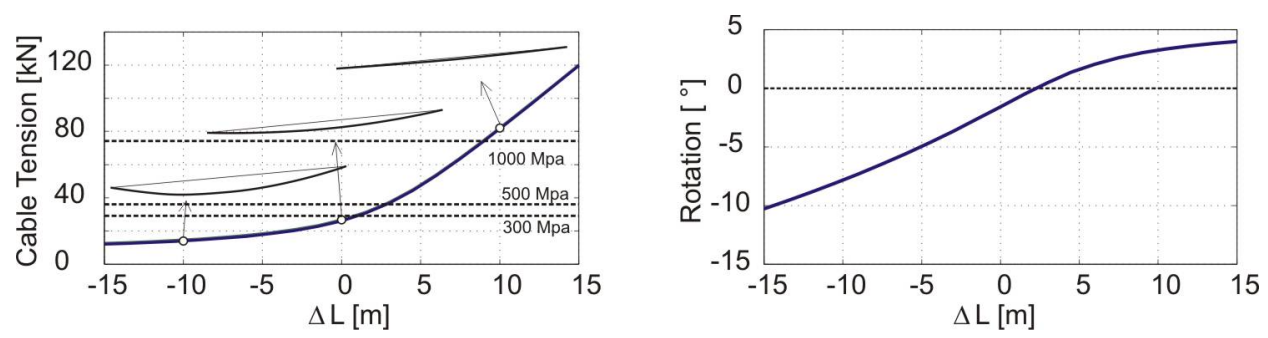

Figure 4: Intial tension vs shortening of the cable chord, and inclination of the lower anchorage.

\section{Results}

Table 1 summarizes the numerical values considered for the problem. The vertical and horizontal offsets $\Delta x$ and $\Delta y$ are determined from topological data. The damping coefficient $\xi$, set equal to $0.1 \%$ by comparison with former identification tests of similar cables, is used to construct the Rayleigh damping matrix. The cable cross section $\Omega$ and Young modulus $E$ are obtained from the specification sheets of the cable. The traveling mass is assumed to be equal to $M_{t}=120 \mathrm{~kg}$, which, according to the fire safety brigade, should correspond to a safe estimation of the body mass, plus equipment. The aerodynamic coefficient $c_{w}$ is tuned in such a way that the results obtained with this model reproduce accurately the travel duration in a 550-m long death-ride which was formerly instrumented.

A number of 16 finite elements is considered throughout this paper. Figure 4 represents the results of the numerical tensioning operation. More precisely, the tension and the inclination at the bottom anchorage are represented in the cable as a function of the imposed kinematic chord extension $\Delta L$. Figure 4 -a indicates that the stress level in the cable, due to its own weight and initial tension, is rather large compared to the ultimate stress of the cable. For instance, stresses as large as $500 \mathrm{MPa}$ correspond to a loose cable, while $1000 \mathrm{MPa}$ at least are necessary to ensure a full stiffness. Figure 4-b is interesting in the sense that, as will be demonstrated clearly later, the inclination of the bottom end of the cable plays an important role in any preliminary design. In this respect, it is important to notice that, to a chord extension of $\Delta L \simeq 2.5 \mathrm{~m}$, corresponds a more or less horizontal inclination of the static deflection of the cable, at the bottom anchorage. In order to allow for a safety margin with respect to the axial force under the riding operations, it is decided to limit the cable extension to $\Delta L=4 \mathrm{~m}$, so that the initial stress in the cable remains below $600 \mathrm{MPa}$ in the static configuration.

The dynamic computation is performed with a constant time step $\Delta t=0.02 \mathrm{~s}$. In order to illustrate the influence of the initial tension in the cable, the dynamic analysis during the ride is performed for three different values of the initial tension in the cable $H=21.7 \mathrm{kN}$ $(\Delta L=-2 m), H=32.4 \mathrm{kN}(\Delta L=2 m)$ and $H=41.6 \mathrm{kN}(\Delta L=4 m)$. The bottom rotation 


\begin{tabular}{|c|c|c|c|c|}
\hline & & $\Delta L=-2 m$ & $\Delta L=2 m$ & $\Delta L=4 m$ \\
\hline$H$ & {$[\mathrm{kN}]$} & 21.7 & 32.4 & 41.6 \\
\hline$\varphi_{0}$ & {[]} & -2.97 & -0.16 & -1.09 \\
\hline$f_{1}$ & {$[\mathrm{~Hz}]$} & 0.161 & 0.141 & 0.137 \\
\hline$f_{2}$ & {$[\mathrm{~Hz}]$} & 0.165 & 0.198 & 0.224 \\
\hline$f_{3}$ & {$[\mathrm{~Hz}]$} & 0.248 & 0.297 & 0.334 \\
\hline
\end{tabular}

Table 2: Properties of the cable under different initial tensions.

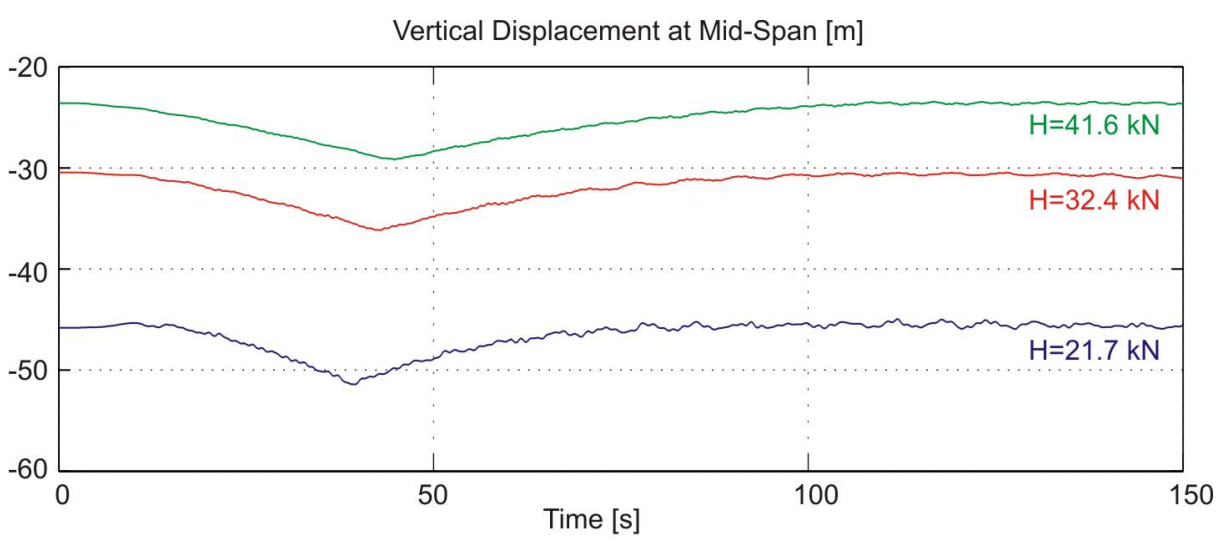

Axial Force at bottom anchorage $[\mathrm{kN}]$

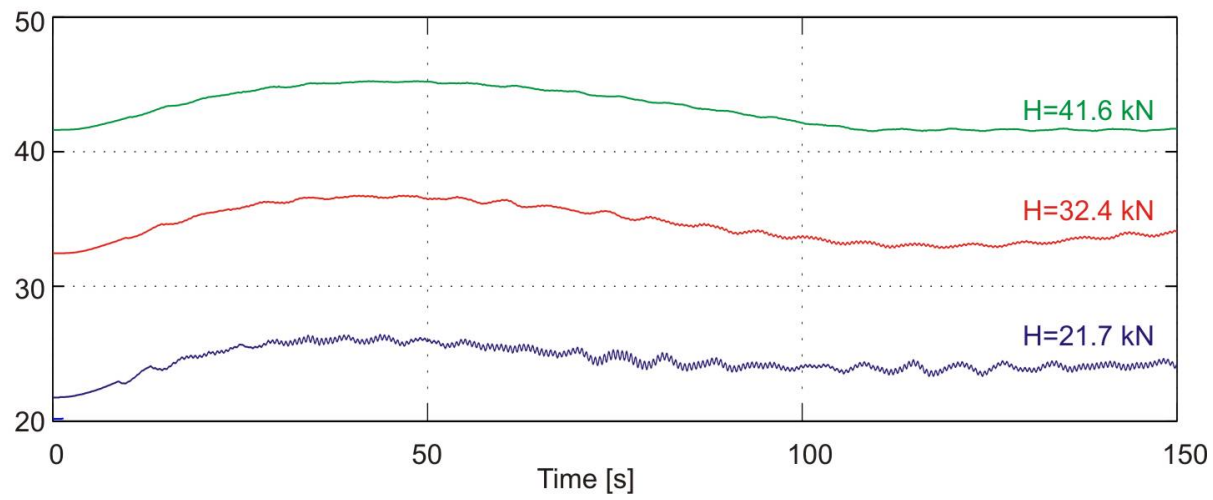

Figure 5: Vertical displacement at mid-span (from chord) and axial force at the bottom. Represented for various initial tension in the cable.

and the natural frequencies of the cable in these various configurations are provided in Table 2 . If the former case, the first two eigen modes have close natural frequencies. This configuration is actually just above the crossover point, as the first mode shape exhibits two halfwaves, while the second one features only one.

Figure 5 represents the vertical displacement at mid-span as well as the axial force in the cable at the bottom anchorage. The static deflection of the loosest cable may reach $45 \mathrm{~m}$ in the static case, plus an additional 10 meters in the dynamic case. For the first two cases $(H=$ $21.7 k N, H=32.4 k N$ ), the cable is loosely taut, which results in (i) a more important increase of the tension force during the ride, (ii) a more flexible, and more oscillating cable. At the opposite, in the case with the highest axial force, the residual oscillations after the ride are damped out by the system. 

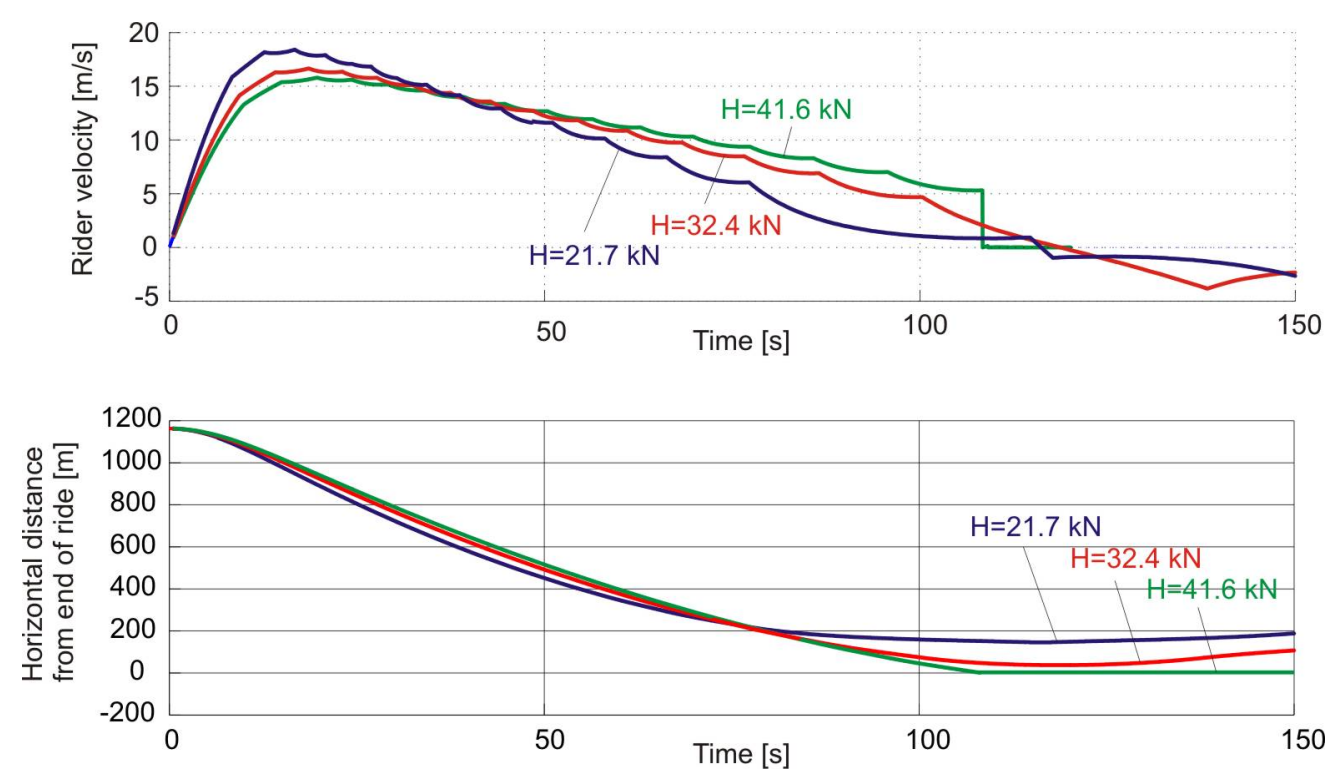

Figure 6: Velocity and vertical position of the rider for various initial tension in the cable.

Figure 6 -a shows that the rider velocity computed with this simplistic model varies with some irregularities, in particular angular points, corresponding to discontinuities in the acceleration. This numerical inaccuracy results from the approximation of the smooth cable by a chain, i.e. a connection of articulated truss elements. At the beginning of the ride, the highest initial tension in the cable $(H=41.6 k N)$ provides a smaller slope in the velocity history, i.e. a smaller acceleration than in the loose cable case. This is justified by the shape of the cable in the neighborhood of the origin, which is flatter for the cable with the highest initial tension. On the contrary, after the rider velocity has reached its maximum value which amounts to $15-20 \mathrm{~m} / \mathrm{s}$ in this particular case study, he/she travels faster when the cable is more tightened. Indeed, the smaller deceleration is attributable to the fact that the rider travels in a steeper zone of the cable.

Figure 6-b represents the horizontal distance between the rider and the lower anchorage. At first sight, there are few differences between the trajectories in the three considered cases, but in fact, they correspond to three significantly different solutions.

The slack cable $(H=21.7 \mathrm{kN})$ has a steeper slope at the beginning, which causes the vertical position of the rider to decrease faster than for the other cases. As the ride goes on, the velocity decreases however rapidly and eventually the rider remains stuck in a sagging area above the River Meuse, more than $150 \mathrm{~m}$ away from the end. This explains why the rider velocity becomes negative after approximately 115 seconds.

The second case, with the intermediate tension in the cable $(H=32.4 \mathrm{kN})$ was actually considered to be optimum. Indeed, the ride ends after 120 seconds with a small rider velocity, which makes it easier to stop him/her, if necessary. Interestingly perhaps, this second case corresponds to a more or less horizontal cable slope at the lower anchorage.

The third case, with the largest initial tension in the cable $(H=41.6 \mathrm{kN})$ was judged to be unsafe as the end velocity was still larger than $5 \mathrm{~m} / \mathrm{s}$ at the end of the ride. Also, starting with an initial horizontal tension in the cable of 4.2 tons was judged to provide stresses in the cable during the dynamic loading that could be to close to the yield limit, with an acceptable safety margin though.

The plots in Figs 5-6 are the perfect tools to judge how large should the initial tension in the cable be. They contrast all aspects related to the decision making process: safety through the 
determination of stresses, sensation through maximal velocity and initial acceleration, comfort through end velocity, feasibility through the horizontal distance from end. The optimal choice should also include considerations on the sensitivity to the rider's mass, i.e. how much these plots change under small or moderate perturbations of the rider's mass.

\section{Conclusions}

This case study on a world record death-ride was here reported with a set of thoughts and design criteria that were considered in the design of this remarkable structure, in particular regarding the proper selection of the initial tension in the cable. A simple numerical model of the problem was built as the connection of two basic features: a finite element model, constructed with 2-D large displacement truss elements in a corotational framework, and a simple staggered coupling model so as to determine the rider's position and velocity.

A parametric study on the initial tension revealed that there exists an optimal tuning of this tension that apparently provides simultaneously comfort, sensation and safety. This solution consists in selecting the initial tension in the cable in such a way that the static configuration of the cable before the ride presents a horizontal inclination at the lower anchorage. As simple as it is, this observation could most likely be extended to other death-rides.

\section{REFERENCES}

[1] Canor T., Denoël V., Vibration analysis of a cable with bending stiffness by a multiple scales approach, paper 292, SEMC 2013, Cape Town, South Africa 6 p. (2013).

[2] Carassale, L., Piccardo, G., Non-linear discrete models for the stochastic analysis of cables in turbulent wind, International Journal of Non-Linear Mechanics, 45 (3), 219-231, 2010.

[3] Denoël V., Detournay E., Multiple scales solution for a beam with a small bending stiffness, ASCE Journal of Engineering Mechanics, 2010. Vol. 136, 69-77, 2012.

[4] Denoël V., Detournay E., Eulerian formulation of constrained elastica, International Journal of Solids and Structures, 48 (3-4), 625-636, 2011.

[5] Denoël V., Canor T., Patching asymptotics solution for a beam with a small bending stiffness, Journal of Structural Engineering, ASCE, 139(2), 180-187, 2012. DOI: $10.1061 /($ asce)st.1943-541x.0000643.

[6] Fryba L., Vibration of Solids and Structures under Moving Loads, Thomas Telford, New York, 1999.

[7] Impollonia, N., Ricciardi, G., Saitta, F., Vibrations of inclined cables under skew wind, International Journal of Non-Linear Mechanics, 46 (7), 907-918, 2011.

[8] Irvine, H. M., Statics of suspended cables, Journal of the Engineering Mechanics Division-Asce, 101 (3), 187-205, 1975.

[9] Irvine H. M., Caughey T. K., The linear theory of free vibrations of a suspended cable, Proceedings of the Royal Society of London, Series A, 314, 299-315, 1974. 
[10] Kevorkian J., Cole J. .D., Multiple Scale and Perturbation Methods, New York: Springer-Verlag, Inc, 1996.

[11] Rega, G., Nonlinear dynamics of suspended cables. Part I : Modeling and analysis; Part II : Deterministic phenomena, ASME Applied Mechanics Reviews, Vol. 57, 443-514, 2004.

[12] Stump, D. M. and van der Heijden, G. H. M., Matched asymptotic expansions for bent and twisted rods: applications for cable and pipeline laying, Journal of Engineering Mathematics, 38 (1), 13-31, 2000.

[13] www.grimpday.com

[14] Zienkievic O. C. , The finite element method in engineering, Pergamon Press, Oxford, 1982. 